\title{
Blueberries inhibit cyclooxygenase-1 and cyclooxygenase-2 activity in human epithelial ovarian cancer
}

\author{
WUMEI LIN and ZHIGANG LI \\ Department of Gynecology, Guangdong General Hospital, Guangdong Academy of Medical Sciences, \\ Guangzhou, Guangdong 510080, P.R. China
}

Received July 30, 2015; Accepted October 19, 2016

DOI: $10.3892 / \mathrm{ol} .2017 .6094$

\begin{abstract}
Ovarian cancer (OC) is the sixth and eighth leading cause of cancer mortality among women in developed and developing countries, respectively. Medical therapy is the main method for the treatment of OC. However, drug toxicity and the marked side effects of chemotherapy limit the usage and therapeutic results of the treatments. Therefore, the identification of multi-target agents with few side effects and high effectiveness is required. Traditional Chinese medicine has been used clinically to treat various types of cancer for thousands of years and is considered to possess multiple components and agents, which exert efficient therapeutic functions with few side effects. Although blueberries have previously been used to treat various types of cancer, the effect on OC and precise molecular mechanism of function of the fruit remains unknown. Cyclooxygenase (COX)-1 and COX-2 have been reported to be the biomarkers of OC. Blueberries may affect the progression of OC by affecting COX levels. To investigate the issue, COX-1 and COX-2 were overexpressed or silenced in ovarian cancer SKOV3 cells. The effect of blueberries on SKOV3 cell viability was determined by an MTT assay. Furthermore, a mouse model for OC was established. The results indicated that blueberries inhibited the proliferation of OC cells by downregulating the levels of COX-1 and COX-2. Blueberry (400 mg daily) consumption reduced tumor size significantly in mice with OC compared with the control without blueberry treatment $(\mathrm{P}<0.05)$. The results suggest that blueberries should be used to develop a potential non-pharmaceutical therapy for OC.
\end{abstract}

\section{Introduction}

Ovarian cancer (OC) begins in the ovary and can result in abnormal cells invading numerous other parts of the body.

Correspondence to: Dr Zhigang Li, Department of Gynecology, Guangdong General Hospital, Guangdong Academy of Medical Sciences, 106 Zhongshan 2nd Road, Guangzhou, Guangdong 510080, P.R. China

E-mail: lizg_fg43@sina.com

Key words: cyclooxygenase-1, cyclooxygenase-2, ovarian cancer, blueberry, mouse model
Symptoms are often vague and unapparent when the disorder begins. There are numerous symptoms for $\mathrm{OC}$, such as bloating (1), pelvic pain (2) and abdominal swelling (3). A number of areas of the body can be affected by OC by the metastasis of abnormal cells, including the abdomen (4), bowel (5), bladder (6), lymph nodes (7) and liver (8). In 2012, there were 239,000 females affected by OC, which caused 152,000 mortalities worldwide (9). OC is the sixth and eighth leading cause of cancer mortality among women in developed and developing countries, respectively (10).

Medical therapy is the most widely used type of treatment for OC; examples include olaparib a biological therapy agent and taxol a chemotherapy agent (10-13). However, the majority of medicines used exhibit unwanted cell toxicity (14-17). Chemotherapy can cause different side effects, such as nausea and vomiting, distress, sexual dysfunction, fatigue and memory loss (18). The toxicity and side effects limit the usage and effectiveness of the aforementioned treatments for OC. It is therefore necessary to find an effective therapeutic method with fewer side effects for OC. Non-pharmaceutical treatment has become a novel therapy for various types of cancer. Blueberries (Vaccinium spp.), a type of fruit, have been identified to exhibit therapeutic effects on several types of cancer $(19,20)$. For example, blueberries inhibit the progression of triple negative breast cancer and triple negative breast cancer-associated metastasis by the inhibition of inflammation via specific cytokine-mediated signaling pathways (21). Blueberries have been revealed to suppress tumor growth and metastasis (21), and are becoming an important fruit due to the associated chemopreventative and therapeutic potential against the tumorigenesis of numerous types of cancer. A blueberry-supplemented diet protects against $17 \beta$-estradiol-mediated mammary tumorigenesis (22). However, the molecular mechanisms of blueberries with respect to the inhibition of various types of cancer are unclear, and the therapeutic effect on OC remains unknown.

Cyclooxygenase (COX), termed prostaglandin-endoperoxide synthase (23), is an enzyme responsible for the formation of prostaglandins. A previous study reported the presence of high levels of COX-1 mRNA in high-grade serous ovarian cancer (24). COX-2 is also overexpressed in human ovarian cancer cells, and targeting COX-2 may be a potential approach for the treatment of OC (25). Blueberries may exhibit a therapeutic effect on $\mathrm{OC}$, and COX-1 and COX-2 may be involved in the process. The present study aimed to explore 
the protective effect of a blueberry diet against the development of OC, which is hypothesized to act via the regulation of COX-mediated pathways.

\section{Materials and methods}

Materials. The blueberries were purchased from the Blueberry Production Field (Guizhou, China). The blueberries were frozen upon arrival at $-20^{\circ} \mathrm{C}$. A total of $100 \mathrm{~g}$ blueberries were homogenized in a homogenizer (GYB60-65; Shanghai Donghua High Pressure Homogenizer Factory, Shanghai, China). The blueberry juice was obtained by centrifuge at $10,000 \mathrm{x} \mathrm{g}, 40^{\circ} \mathrm{C}$ for $10 \mathrm{~min}(1 \mathrm{ml}$ blueberry juice was produced from $2 \mathrm{~g}$ blueberry).

Cell culture. The human ovarian cancer SKOV3 cell line was purchased from the Type Culture Collection of the Chinese Academy of Sciences (Shanghai, China) and cultured with McCoy's 5A medium without serum (Invitrogen; Thermo Fisher Scientific, Inc., Waltham, MA, USA). The medium was supplemented with $4 \mu \mathrm{g} / \mathrm{ml}$ transferrin (Chemicon International Inc.; EMD Millipore, Billerica, MA, USA), $5 \mu \mathrm{g} / \mathrm{ml}$ insulin (Sigma-Aldrich, St. Louis, MO, USA). and $10 \mathrm{ng} / \mathrm{ml}$ vascular endothelial growth factor (Chemicon International Inc.; EMD Millipore). The cells were cultured in $5 \% \mathrm{CO}_{2}$ at $37^{\circ} \mathrm{C}$ and $100 \%$ humidity, and transferred to fresh medium every 2 days.

Evaluation of cell viability by MTT assay. Cell viability was determined using an MTT assay. The SKOV3 cells were seeded into 96-well plates at a concentration of $1 \times 10^{4}$ cells per well in $100 \mu \mathrm{l} \mathrm{McCoy's} \mathrm{5A} \mathrm{medium.} \mathrm{The} \mathrm{cells} \mathrm{were} \mathrm{treated} \mathrm{for}$ 24,48 or $72 \mathrm{~h}$ with $0,1,2,4,8$ or $16 \mathrm{mg} / \mathrm{ml}$ blueberry juice. At the end of the culture, $5 \mu 1 \mathrm{MTT}(10 \mathrm{mg} / \mathrm{ml}$ in PBS) was added to each well and incubated for an additional $3 \mathrm{~h}$ at room temperature. Untreated cells were used as control groups. The purple formazan was dissolved in dimethyl sulfoxide. Absorbance was recorded at $570 \mathrm{~nm}$ using an ELISA reader (Elisa Reader KD600; Shanghai Tongge Medical Devices Co., Ltd., Shanghai, China).

COX-1 and COX-2 expression construction. COX-1 and COX-2 genes were amplified and cloned into the Nhel-EcoRI sites of pcDNA3.1 plasmid (Invitrogen; Thermo Fisher Scientific, Inc.), which were termed pcDNA3.1-COX-1 and pcDNA3.1-COX-2. The details of the CPX primers are as follows: COX-1 accession number, BC029840.1, sense, 5'-GTGAGCTAGCATGAGCCGGAGTCTCTTGC-3' and antisense primers, 5'-CTGAGAATTCTCAGAGCTCTGTGG ATGGTC-3', generating a 1820-base pair (bp) product; COX-2 accession number, AY462100.1, sense, 5'-GTGAGCTAGCAT GCTCGCCCGCGCCCTGC-3' and antisense primers, 5'-CTG AGAATTCCTACAGTTCAGTCGAACGTTC-3', generating an 1835-bp product. The constructed plasmids were amplified in Escherichia coli (Takara Biotechnology Co., Ltd., Dalian, China), isolated using a plasmid Miniprep Kit (Beijing TransGen Biotech Co., Ltd., Beijing, China) and were verified by sequencing (Takara Biotechnology Co., Ltd.). The classical Sanger sequencing was used. PCR reaction mixture was prepared using the following: $1 \mu 1100 \mathrm{ng}$ plasmid, $1 \mu \mathrm{l}$ primer (5 pmol; 5' Sequencing 1 Primer, CMV-F; 3' Sequencing
1 Primer, BGH-rev), $2 \mu 1$ BigDye, $4 \mu 1$ 5X buffer, $0.5 \mu 1$ Taq DNA polymerase and $\mathrm{ddH}_{2} \mathrm{O}$ added to $20 \mu \mathrm{l}$. The PCR reaction begins at $95^{\circ} \mathrm{C} 1 \mathrm{~min}$, then 35 cycles of $96^{\circ} \mathrm{C} 10 \mathrm{sec}, 52^{\circ} \mathrm{C}$ $15 \mathrm{sec}$ and $72^{\circ} \mathrm{C} 1 \mathrm{~min}$.

COX-1 and COX-2 RNAi. The lentivector pLKO.1 1 was purchased from Academia Sinica (Taipei, Taiwan). The siRNA sequence targeting COX-1, 5'-GTGAGCTATTACACT CGTATT-3' and COX-2, 5'-GATTGACAGTCCACCAAC TTA-3' was synthesized by Takara Biotechnology Co., Ltd., and linked to the lentivector pLKO.1, and therefore termed pLKO.1-COX-1-siRNA and pLKO.1-COX-2-siRNA. The SKOV3 cells were cotransfected by the reconstructed vectors by Lipofectamine ${ }^{\circledR} 2000$ (Shanghai Yijie Biotechnology Co., Ltd., Shanghai, China) and pPACK Packaging Plasmid Mix (System Biosciences, Palo Alto, CA, USA).

Orthotopic implantation. All procedures were approved by the Animal Care and Ethics Committee of Guangdong General Hospital (Guangzhou, China). A total of 40 BALB/c 3-week-old nude mice (male:female $=1: 1 ; 15-20 \mathrm{~g}$; height, 2-2.5 cm) purchased from the Animal Center of Guangdong Academy of Medical Sciences (Guangzhou, China) were raised in a high-efficiency particulate arrestance-filtered environment with a 12-h light/dark cycle. The SKOV3 cells were subcutaneously injected into 32 nude mice. At $\sim 1 \mathrm{~cm}^{3}$, the xenograft was excised and minced, and implanted into other 4-week old BALB/c nude mice according to the protocol of a previous study (26). A total of 4 days subsequent to the implantation of the OC tumors, the effects of blueberries on the mice was tested.

Experimental design. All the mice were assigned into either control or model group, with the model group being administered different concentrations of blueberry juice (100, 200 and $400 \mathrm{mg}$ daily). The control and model groups were fed a normal diet and water, and all the model groups were administered a normal diet plus the corresponding blueberry juice. Subsequent to 2 weeks, the mice were sacrificed via cervical dislocation. Tumors were isolated under sterile conditions on a nutrient culture medium. Tumor size was determined using the ellipsoid formula $v=4 / 3 \pi$ abc, where $v$ is volume, a and $c$ are equal to half tumor height, and $b$ is half tumor length. All the procedures for animal studies were consistent with the Animal Care and Use Guidance of Guangdong Academy of Medical Sciences (Guangzhou, China).

Quantitative reverse transcriptase-polymerase chain reaction (RT-qPCR). Total RNA was purified from ovarian tumors of animal models or healthy mice using an RNA purification kit (cat. no. 74106; Qiagen, Inc., Valencia, CA, USA). Tumor tissues were cut into slices $<2 \mathrm{~mm}$ and homogenized in $500 \mu \mathrm{l}$ TRIzol. Phase was separated by the addition of $500 \mu 1$ chloroform, and centrifuged at 12,000 x g for $10 \mathrm{~min}$. The RNA was precipitated by adding $500 \mu 1$ isopropyl alcohol. RNA was purified using a purification column. A total of $1 \mu \mathrm{g}$ RNA from each sample was reverse-transcribed using the High-Capacity cDNA Reverse Transcription kit (cat. no. 4368813; Thermo Fisher Scientific, Inc., Carlsbad, CA, USA). A total of $1 \mu 1$ purified RNA, poly(A)+-selected RNA primed with oligo 


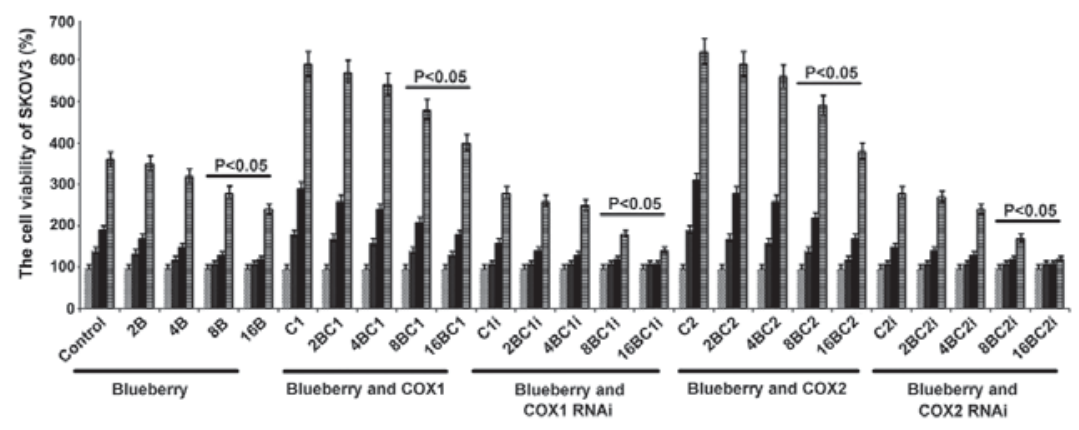

Figure 1. Blueberries inhibit cell growth rate of SKOV3 cells. SKOV3 cells were transfected with COX-1, COX-2, COX-1 RNAi and COX-2 RNAi, and treated with $0,2,4,8$ and $16 \mathrm{mg} / \mathrm{ml}$ blueberry juice. Cell concentrations were determined using MTT assays at days $0,1,2$ and 3 . The data represent the results of 5 independent experiments and are presented as the mean \pm standard deviation. $\mathrm{P}<0.05$ compared with $0 \mu \mathrm{g} / \mathrm{ml}$ blueberry subsequent to a 1-, 2- or 3-day culture. COX, cyclooxygenase; RNAi, RNA interference; Control, without transfection or blueberry; 2B, 2 mg blueberry; 4B, 4 mg blueberry; 8B, 8 mg blueberry; 16B, 16 mg blueberry; C1, COX-1; 2BC1, 2 mg blueberry and COX1 transfection; 4BC1, $4 \mathrm{mg}$ blueberry and COX1 transfection; 8BC1, $8 \mathrm{mg}$ blueberry and COX1 transfection; 16BC1, $16 \mathrm{mg}$ blueberry and COX1 transfection; C1i, COX-1 RNAi; 2BCli, 2 mg blueberry and COX1 RNAi; 4BC1i, $4 \mathrm{mg}$ blueberry and COX1 RNAi; 8BC1i, $8 \mathrm{mg}$ blueberry and COX1 RNAi; 16BCli, $16 \mathrm{mg}$ blueberry and COX1 RNAi; C2, COX-2; 2BC2, 2 mg blueberry and COX2 transfection; 4BC2, $4 \mathrm{mg}$ blueberry and COX2 transfection; 8BC2, $8 \mathrm{mg}$ blueberry and COX2 transfection; 16BC2, $16 \mathrm{mg}$ blueberry and COX2 transfection; C2i, COX-2 RNAi; 2BC2i, $2 \mathrm{mg}$ blueberry and COX2 RNAi; 4BC2i, $4 \mathrm{mg}$ blueberry and COX2 RNAi; 8BC2i, 8 mg blueberry and COX2 RNAi; $16 \mathrm{BC} 2 \mathrm{i}, 16 \mathrm{mg}$ blueberry and COX2 RNAi.

(deoxy-thymidine) $100 \mathrm{nM}, 1 \mathrm{X}$ annealing buffer and water were heated to $65^{\circ} \mathrm{C}$ for $5 \mathrm{~min}$ and placed on ice for $1 \mathrm{~min}$. This reaction mix and $0.5 \mu 1$ reverse transcriptase were added to the reaction for a final volume of $40 \mu \mathrm{l}$, and the mixture was incubated at $50^{\circ} \mathrm{C}$ for $50 \mathrm{~min}$. The mixture was then heated to $95^{\circ} \mathrm{C}$ for 5. The SYBR Green DNA PCR kit was purchased from Applied Biosystems (Thermo Fisher Scientific, Inc.) and used for RT-qPCR using Applied Biosystems ${ }^{\circledR} 7500$ Real-Time PCR Systems (Thermo Fisher Scientific, Inc.). The PCR primers were as follows: COX-1 forward, 5'-CAGAGCCAGATGGCT GTGGG-3' and reverse, 5'-AAGCTGCTCATCGCCCCAG G-3'; COX-2 forward, 5'-AAGTGCGATTGTACCCGGAC-3' and reverse, 5'-ACGTTCCAAAATCCCTTGAA-3'; GAPDH forward, 5'-AACTACATGGTTTACATGTT-3' and reverse, 5'-CACTTGATTTTGGAGGGATC-3'. After 1 min enzyme activation at $95^{\circ} \mathrm{C}$, the reaction was cycled 45 times at $95^{\circ} \mathrm{C}$ for $10 \mathrm{~s}, 55^{\circ} \mathrm{C}$ for $10 \mathrm{~s}$, and $72^{\circ} \mathrm{C}$ for $20 \mathrm{~s}$. The values of the target genes were calculated utilizing the $2^{-\Delta \Delta \mathrm{Cq}}$ method (27) for the fold change compared with GAPDH. The mRNA levels of the target genes were presented as relative increases compared with GAPDH. All experiments were performed in triplicate.

ELISA analysis. The concentrations of COX-1 and COX-2 in the treated and untreated samples were measured using Human COX-1 ELISA kit (cat. no. MBS026381) and Human COX-2 ELISA kit (cat. no. MBS043833; MyBioSource, San Diego, CA, USA), respectively.

Statistical analysis. Data were analyzed using one-way analysis of variance in SPSS 20 (IBM SPSS, Armonk, NY, USA). Quantitative data are shown as the mean \pm standard deviation. $\mathrm{P}<0.05$ was considered to indicate a statistically significant difference.

\section{Results}

Effect of blueberry on the growth of SKOV3 cells. To investigate the effects of blueberries on SKOV3 cells, the concentration-dependent effects of blueberry juice was explored. It was found that blueberries inhibited cell growth in a dose-dependent way. Subsequent to the exposure of SKOV3 cells to $16 \mathrm{mg} / \mathrm{ml}$ blueberry, the cell growth rate was reduced by up to $28 \%$ compared with the control following the 3-day culture ( $\mathrm{P}=0.018$; Fig. 1). Based on these results, the present study used a higher blueberry concentration $(16 \mathrm{mg} / \mathrm{ml})$ to treat the OC mouse models (400 mg daily for a $25 \mathrm{~g}$ mouse).

Effects of COX-1 and COX-2 on the growth rate of SKOV3 cells. To investigate the effects of COX-1 and COX-2 on SKOV3 cells, the genes were overexpressed by gene transformation or inhibited by RNAi in the cells. Exposure of SKOV3 cells to $16 \mathrm{mg} / \mathrm{l}$ blueberry resulted in a reduction of cell concentration detected by MTT when compared with the control $(\mathrm{P}=0.019$; Fig. 1$)$. When $\mathrm{COX}-1$ or $\mathrm{COX}-2$ was inhibited by RNAi in SKOV3 cells and treated with $16 \mathrm{mg} / \mathrm{ml}$ blueberry juice, the growth rate of the cells was reduced by up to $50 \%$ compared with the growth rate of the control group subsequent to $72 \mathrm{~h}$ culture (Fig. 1). By contrast, if COX-1 or COX-2 was overexpressed in SKOV3 cells, the growth rate increased by up to $66 \%$ compared with the growth rate of the control group subsequent to $72 \mathrm{~h}$ culture (Fig. 1). The increase was inhibited completely when $16 \mathrm{mg} / \mathrm{ml}$ blueberry juice was added to the cells.

mRNA level of COX-1 and COX-2 in SKOV3. The mRNA level of COX-1 was significantly affected by blueberry juice and was reduced by up to $50 \%$ when $16 \mathrm{mg} / \mathrm{ml}$ blueberry juice was added compared with the control without the blueberry treatment ( $\mathrm{P}=0.002$; Fig. 2). The mRNA level of COX-1 increased by up to $50 \%$ when SKOV3 cells were transfected with COX-1, compared with the control without COX-1 transfection ( $\mathrm{P}=0.002$; Fig. 2). The increase was inhibited by the addition of blueberry juice in a dose-dependent way. The mRNA level of COX-1 was reduced to 0 when SKOV3 cells were transfected with COX-1 RNAi (Fig. 2). Neither the overexpression nor gene silencing of COX-2 affected the mRNA level of COX-1 ( $\mathrm{P}=0.235$; Fig. 2). 


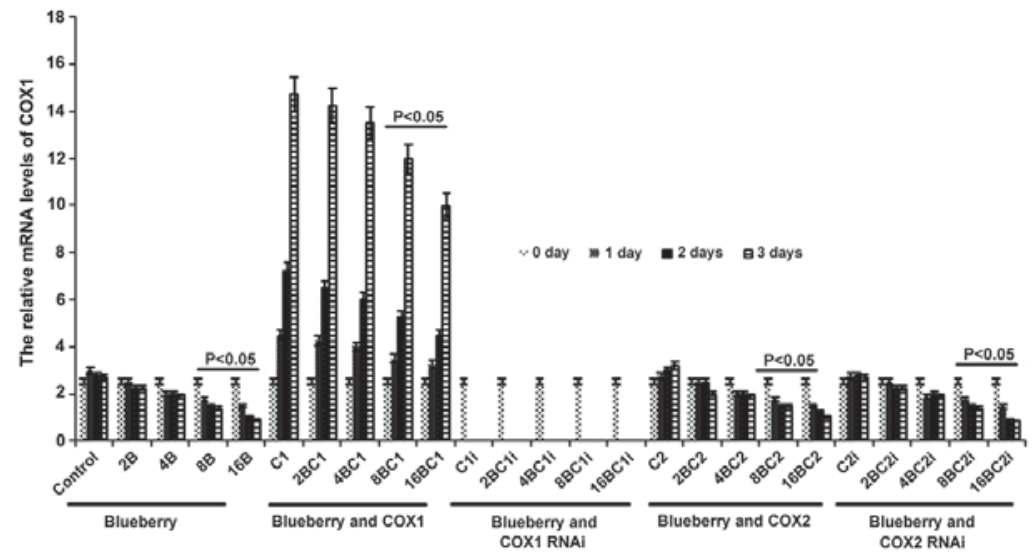

Figure 2. Reverse transcription-qualitative polymerase chain reaction analyses show that blueberries reduce relative mRNA levels of COX-1 in SKOV3 cells SKOV3 cells were transfected with COX-1, COX-2, COX-1 RNAi or COX-2 RNAi, and treated with $0,2,4,8$ or $16 \mathrm{mg} / \mathrm{ml}$ blueberry juice. The relative mRNA levels of COX-1 were measured at days 0,1,2 and 3. GAPDH was used as an internal control. The data represent the results of 5 independent experiments and are presented as the mean \pm standard deviation. $\mathrm{P}<0.05$ compared with $0 \mu \mathrm{g} / \mathrm{ml}$ blueberry subsequent to a 1-, 2- or 3-day culture. COX, cyclooxygenase; RNAi, RNA interference; Control, without transfection or blueberry; 2B, $2 \mathrm{mg}$ blueberry; 4B, $4 \mathrm{mg}$ blueberry; 8B, $8 \mathrm{mg}$ blueberry; 16B, $16 \mathrm{mg}$ blueberry; C1, COX-1; 2BC1, 2 mg blueberry and COX1 transfection; 4BC1, 4 mg blueberry and COX1 transfection; 8BC1, 8 mg blueberry and COX1 transfection; 16BC1, $16 \mathrm{mg}$ blueberry and COX1 transfection; Cli, COX-1 RNAi; 2BCli, $2 \mathrm{mg}$ blueberry and COX1 RNAi; 4BCli, $4 \mathrm{mg}$ blueberry and COX1 RNAi; 8BCli, $8 \mathrm{mg}$ blueberry and COX1 RNAi; 16BC1i, 16 mg blueberry and COX1 RNAi; C2, COX-2; 2BC2, 2 mg blueberry and COX2 transfection; 4BC2, 4 mg blueberry and $\mathrm{COX} 2$ transfection; $8 \mathrm{BC} 2,8 \mathrm{mg}$ blueberry and $\mathrm{COX} 2$ transfection; $16 \mathrm{BC} 2,16 \mathrm{mg}$ blueberry and $\mathrm{COX} 2$ transfection; $\mathrm{C} 2 \mathrm{i}, \mathrm{COX}-2 \mathrm{RNAi} ; 2 \mathrm{BC} 2 \mathrm{i}, 2 \mathrm{mg}$ blueberry and COX2 RNAi; 4BC2i, 4 mg blueberry and COX2 RNAi; 8BC2i, 8 mg blueberry and COX2 RNAi; 16BC2i, 16 mg blueberry and COX2 RNAi.

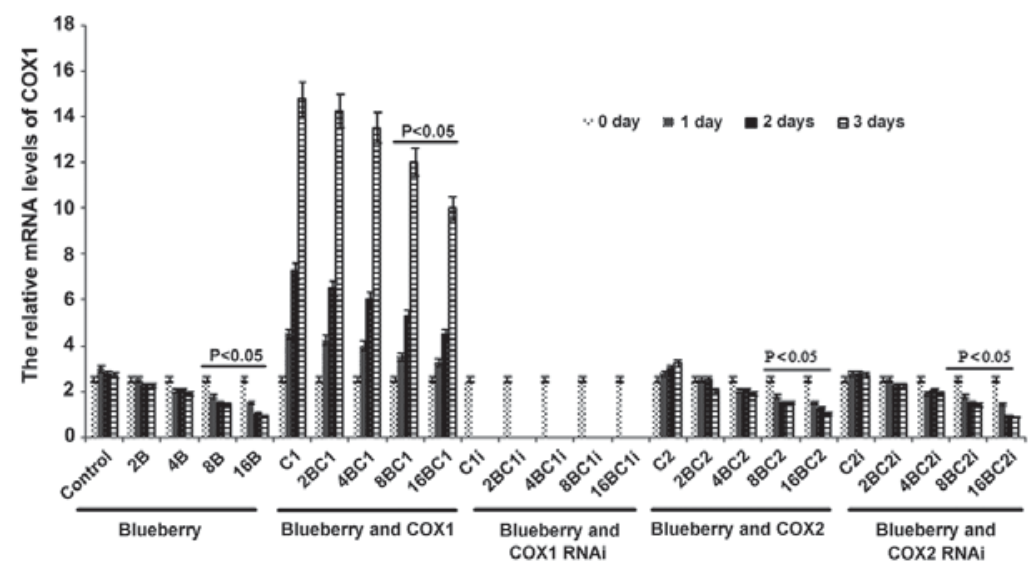

Figure 3. Reverse transcription qualitative-polymerase chain reaction analyses show that blueberries reduce relative mRNA levels of COX-2 in SKOV3 cells. SKOV3 cells were transfected with COX-1, COX-2, COX-1 RNAi or COX-2 RNAi, and treated with $0,2,4,8$ or $16 \mathrm{mg} / \mathrm{ml}$ blueberry juice. The relative mRNA levels of COX-2 were measured at days 0,1,2 and 3. GAPDH was used as an internal control. The data represent the results of 5 independent experiments and are presented as the mean \pm standard deviation. $\mathrm{P}<0.05$ compared with $0 \mu \mathrm{g} / \mathrm{ml}$ blueberry subsequent to a 1-, 2- or 3-day culture. COX, cyclooxygenase; RNAi, RNA interference; Control, without transfection or blueberry; 2B, 2 mg blueberry; 4B, 4 mg blueberry; 8B, 8 mg blueberry; 16B, 16 mg blueberry; C1, COX-1; 2BC1, 2 mg blueberry and COX1 transfection; 4BC1, 4 mg blueberry and COX1 transfection; 8BC1, 8 mg blueberry and COX1 transfection; 16BC1, $16 \mathrm{mg}$ blueberry and COX1 transfection; C1i, COX-1 RNAi; 2BC1i, $2 \mathrm{mg}$ blueberry and COX1 RNAi; 4BC1i, 4 mg blueberry and COX1 RNAi; 8BC1i, 8 mg blueberry and COX1 RNAi; 16BC1i, $16 \mathrm{mg}$ blueberry and COX1 RNAi; C2, COX-2; 2BC2, 2 mg blueberry and COX2 transfection; 4BC2, 4 mg blueberry and COX2 transfection; 8BC2, $8 \mathrm{mg}$ blueberry and COX2 transfection; 16BC2, $16 \mathrm{mg}$ blueberry and COX2 transfection; C2i, COX-2 RNAi; 2BC2i, 2 mg blueberry and COX2 RNAi; 4BC2i, 4 mg blueberry and COX2 RNAi; 8BC2i, 8 mg blueberry and COX2 RNAi; 16BC2i, 16 mg blueberry and COX2 RNAi.

The mRNA level of COX-2 was significantly affected by blueberry juice, decreasing by up to $50 \%$ when $16 \mathrm{mg} / \mathrm{ml}$ blueberry was added compared with the control without the blueberry treatment ( $\mathrm{P}=0.008$; Fig. 3). The mRNA level of COX-2 also increased by up to $150 \%$ when the SKOV3 cells were transfected with COX-2 compared with the control without COX-2 transfection ( $\mathrm{P}=0.002$; Fig. 3$)$. The increase was inhibited by the addition of blueberry juice in a dose-dependent way. The mRNA level of COX-2 decreased to 0 when the SKOV3 cells were transfected with COX-2 RNAi (Fig. 3). Neither the overexpression nor gene silencing of COX-1 affected the mRNA level of COX-2 $(\mathrm{P}=0.321$; Fig. 3).

Levels of COX-1 and COX-2 in SKOV3. The results of the levels of COX-1 and COX-2 in SKOV3 were similar compared with the results from the mRNA levels investigation. The concentration of COX-1 was significantly affected by the blueberry juice, being reduced by up to $50 \%$ when $16 \mathrm{mg} / \mathrm{ml}$ blueberry juice was added, compared with the control without the blueberry treatment ( $\mathrm{P}=0.003$; Fig. 4). The concentration of COX-1 increased by up to $50 \%$ when the SKOV3 cells were 


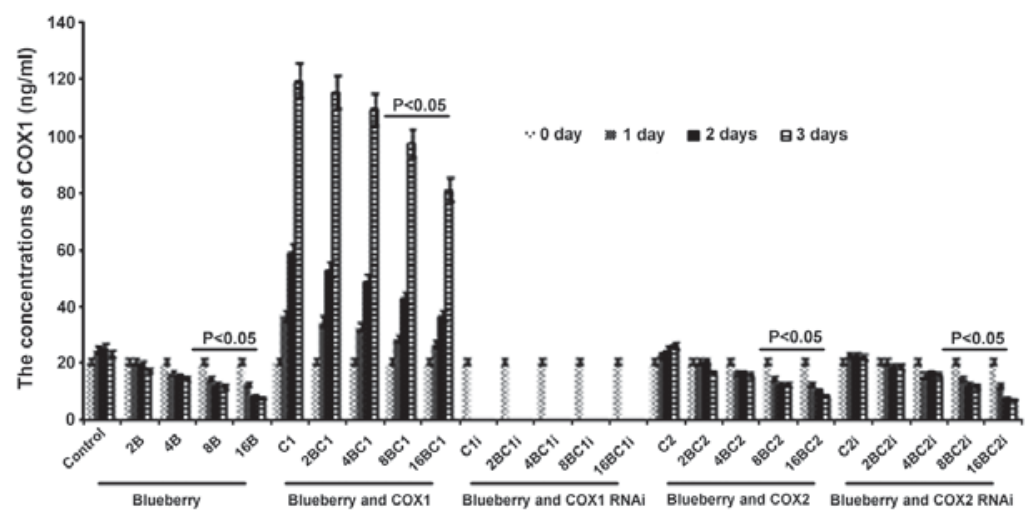

Figure 4. ELISA analyses show that blueberries reduce the protein levels of COX-1 in SKOV3 cells. SKOV3 cells were transfected with COX-1, COX-2, COX-1 RNAi or COX-2 RNAi, and treated with $0,2,4,8$ or $16 \mathrm{mg} / \mathrm{ml}$ blueberry juice. The concentrations of COX-1 were measured at days $0,1,2$ and 3 . The data represent the results of 5 independent experiments and are presented as the mean \pm standard deviation. $\mathrm{P}<0.05$ compared with $0 \mu \mathrm{g} / \mathrm{ml}$ blueberry subsequent to a 1-, 2- or 3-day culture. COX, cyclooxygenase; RNAi, RNA interference; Control, without transfection or blueberry; 2B, 2 mg blueberry; 4B, $4 \mathrm{mg}$ blueberry; 8B, $8 \mathrm{mg}$ blueberry; 16B, 16 mg blueberry; C1, COX-1; 2BC1, 2 mg blueberry and COX1 transfection; 4BC1, 4 mg blueberry and COX1 transfection; 8BC1, $8 \mathrm{mg}$ blueberry and COX1 transfection; 16BC1, $16 \mathrm{mg}$ blueberry and COX1 transfection; Cli, COX-1 RNAi; 2BCli, 2 mg blueberry and COX1 RNAi; 4BC1i, 4 mg blueberry and COX1 RNAi; 8BC1i, 8 mg blueberry and COX1 RNAi; 16BC1i, 16 mg blueberry and COX1 RNAi; C2, COX-2; 2BC2, $2 \mathrm{mg}$ blueberry and COX2 transfection; 4BC2, $4 \mathrm{mg}$ blueberry and COX2 transfection; 8BC2, $8 \mathrm{mg}$ blueberry and COX2 transfection; $16 \mathrm{BC} 2,16 \mathrm{mg}$ blueberry and COX2 transfection; C2i, COX-2 RNAi; 2BC2i, 2 mg blueberry and COX2 RNAi; 4BC2i, 4 mg blueberry and COX2 RNAi; 8BC2i, 8 mg blueberry and COX2 RNAi; 16BC2i, 16 mg blueberry and COX2 RNAi.

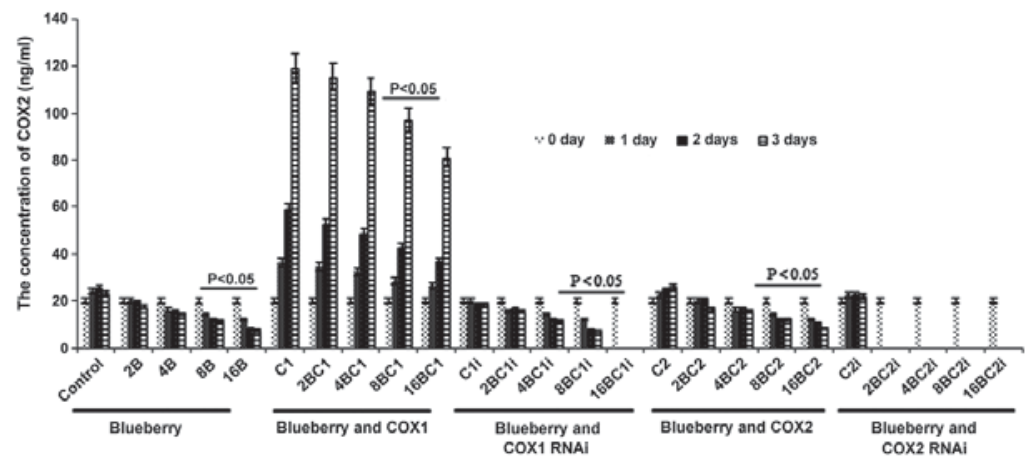

Figure 5. ELISA analyses show that blueberries reduce the protein levels of COX-2 in SKOV3 cells. SKOV3 cells were transfected with COX-1, COX-2, COX-1 RNAi or COX-2 RNAi, and treated with $0,2,4,8$ or $16 \mathrm{mg} / \mathrm{ml}$ blueberry juice. The concentrations of COX-2 were measured at days $0,1,2$ and 3 . The data represents the results of 5 independent experiments and are presented as the mean \pm standard deviation. $\mathrm{P}<0.05$ compared with $0 \mu \mathrm{g} / \mathrm{ml}$ blueberry subsequent to a 1-, 2- or 3-day culture. COX, cyclooxygenase; RNAi, RNA interference; Control, without transfection or blueberry; 2B, $2 \mathrm{mg}$ blueberry; 4B, 4 mg blueberry; 8B, 8 mg blueberry; 16B, 16 mg blueberry; C1, COX-1; 2BC1, 2 mg blueberry and COX1 transfection; 4BC1, 4 mg blueberry and COX1 transfection; 8BC1, $8 \mathrm{mg}$ blueberry and $\mathrm{COX} 1$ transfection; 16BC1, $16 \mathrm{mg}$ blueberry and COX1 transfection; C1i, COX-1 RNAi; 2BCli, $2 \mathrm{mg}$ blueberry and COX1 RNAi; 4BCli, $4 \mathrm{mg}$ blueberry and COX1 RNAi; 8BC1i, 8 mg blueberry and COX1 RNAi; 16BCli, 16 mg blueberry and COX1 RNAi; C2, COX-2; 2BC2, 2 mg blueberry and $\mathrm{COX} 2$ transfection; 4BC2, $4 \mathrm{mg}$ blueberry and $\mathrm{COX} 2$ transfection; $8 \mathrm{BC} 2,8 \mathrm{mg}$ blueberry and $\mathrm{COX} 2$ transfection; $16 \mathrm{BC} 2,16 \mathrm{mg}$ blueberry and COX2 transfection; C2i, COX-2 RNAi; 2BC2i, 2 mg blueberry and COX2 RNAi; 4BC2i, 4 mg blueberry and COX2 RNAi; 8BC2i, 8 mg blueberry and COX2 RNAi; 16BC2i, 16 mg blueberry and COX2 RNAi.

transfected with COX-1, compared with the control without COX-1 transfection ( $\mathrm{P}=0.002$; Fig. 4). The increase was inhibited by the addition of blueberry juice in a dose-dependent way. The concentration of COX-1 decreased to 0 when the SKOV3 cells were transfected with COX-1 RNAi (Fig. 4). Neither the overexpression nor gene silencing of COX-2 affected the concentration of COX-1 ( $\mathrm{P}=0.287$; Fig. 4).

Similarly, the concentration of COX-2 was significantly affected by blueberry juice, being reduced by up to $50 \%$ when $16 \mathrm{mg} / \mathrm{ml}$ blueberry juice was added, compared with the control without the blueberry treatment $(\mathrm{P}=0.002$; Fig. 5). The mRNA level of COX-2 increased by up to $150 \%$ when SKOV3 cells were transfected with COX-2, compared with the control without $\mathrm{COX}-2$ transfection ( $\mathrm{P}=0.003$; Fig. 5). The increase was inhibited by the addition of blueberry juice in a dose-dependent way. The concentration of COX-2 decreased to 0 when the SKOV3 cells were transfected with COX-2 RNAi (Fig. 5). Neither the overexpression nor gene silencing of COX-1 affected the concentration of COX-2 ( $\mathrm{P}=0.329$; Fig. 3).

The aforementioned results suggest that blueberries reduce the concentration of COX-1 and COX-2 in a dose-dependent way. Blueberries inhibit the growth rate of SKOV3 by decreasing the levels of COX-1 and COX-2, while COX-1 and COX-2 promote the growth of SKOV3.

Effects of blueberry on tumor volume. Prior to the blueberry treatment, the volumes of the OC tumors were similar between groups. Subsequent to the blueberry treatment, the volumes of $\mathrm{OC}$ in the $500 \mathrm{mg}$ group significantly decreased by $40 \%$ compared with the group that received no 


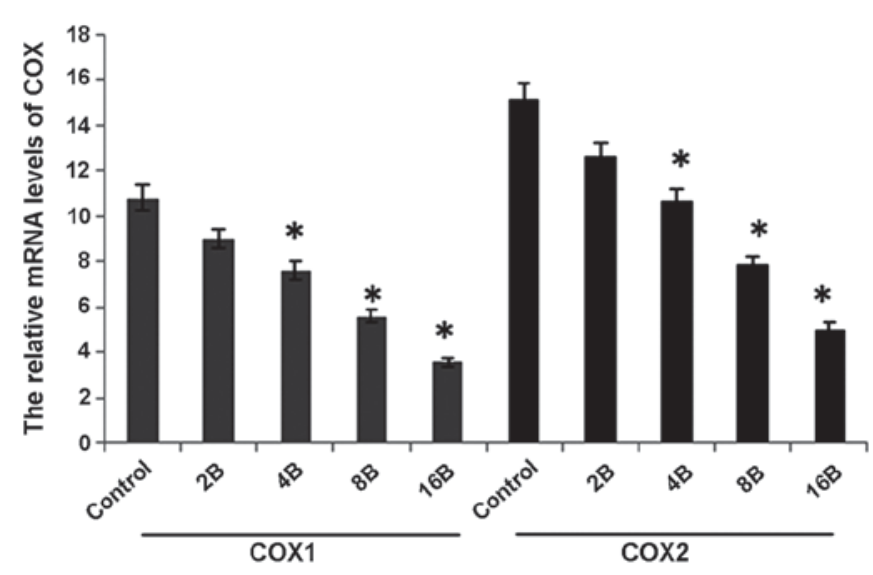

Figure 6. Reverse transcription quantitative-polymerase chain reaction analyses show that blueberries reduce relative mRNA levels of COX-1 and COX-2 of ovarian cancer in a mouse model. The mice were treated with 0,2 , 4,8 or $16 \mathrm{mg} / \mathrm{ml}$ blueberry juice. The relative mRNA levels of COX-1 and COX-2 were measured subsequent to a 14-day culture. GAPDH was used as an internal control. The data represent the results of 5 independent experiments and are presented as the mean \pm standard deviation. ${ }^{*} \mathrm{P}<0.05$ compared with $0 \mu \mathrm{g} / \mathrm{ml}$ blueberry. COX, cyclooxygenase; Control, without transfection or blueberry; 2B, $2 \mathrm{mg}$ blueberry; 4B, $4 \mathrm{mg}$ blueberry; $8 \mathrm{~B}, 8 \mathrm{mg}$ blueberry; $16 \mathrm{~B}, 16 \mathrm{mg}$ blueberry.

blueberry treatment $(\mathrm{P}=0.0008)$. The high-efficiency inhibitory concentrations of blueberry were between 8 and $16 \mathrm{mg}$. The weights of the OC tumors were $4.82 \pm 0.41,4.25 \pm 0.35$, $3.16 \pm 0.23$ and $2.47 \pm 0.26 \mathrm{~g}$ in blank, $0,100,200$ and $400 \mathrm{mg}$ blueberry groups, respectively, yielding growth inhibitions of $12.5,35.4$, and $49.4 \%$. From the aforementioned results, the $400 \mathrm{mg}$ blueberry group showed significant inhibitory results for OC $(\mathrm{P}=0.014)$.

Blueberry juice affects the mRNA levels of COX-1 and COX-2 of $O C$ in the mouse model. The present study investigated the effect of blueberries on the mRNA levels of COX-1 and COX-2 of OC in a mouse model, which are biomarkers of OC $(24,28)$. The mRNA levels of COX-1 and COX-2 of OC in the mice were the highest in the control group compared with those in the models treated with blueberry juice ( $\mathrm{P}=0.006$; Fig. 6). Comparatively, the levels of COX-1 and COX-2 significantly decreased by up to 63.6 and $68.7 \%$, respectively, when the mice were treated with $400 \mathrm{mg}$ blueberry juice daily compared with those from the control without the addition of blueberry juice $(\mathrm{P}=0.002$; Fig. 6). The results suggested that blueberry juice significantly affects the mRNA levels of COX-1 and COX-2 of OC in the mouse model studied.

Blueberry juice affects the levels of COX-1 and COX-2 of OC in the mouse model. In concordance with the mRNA results, ELISA analysis showed higher levels of COX-1 and COX-2 of $\mathrm{OC}$ in mice in the control group when compared with the groups treated with blueberry juice $(\mathrm{P}=0.012$; Fig. 7). Comparatively, the levels of COX-1 and COX-2 significantly decreased by up to 57.1 and $54.5 \%$, respectively, when the mice were treated with $400 \mathrm{mg}$ blueberry juice daily compared with the protein levels of the control without the addition of blueberry juice $(\mathrm{P}=0.006$; Fig. 7). The aforementioned results suggested that blueberry juice significantly affects the levels of COX-1 and COX-2 of OC in the mouse model studied.

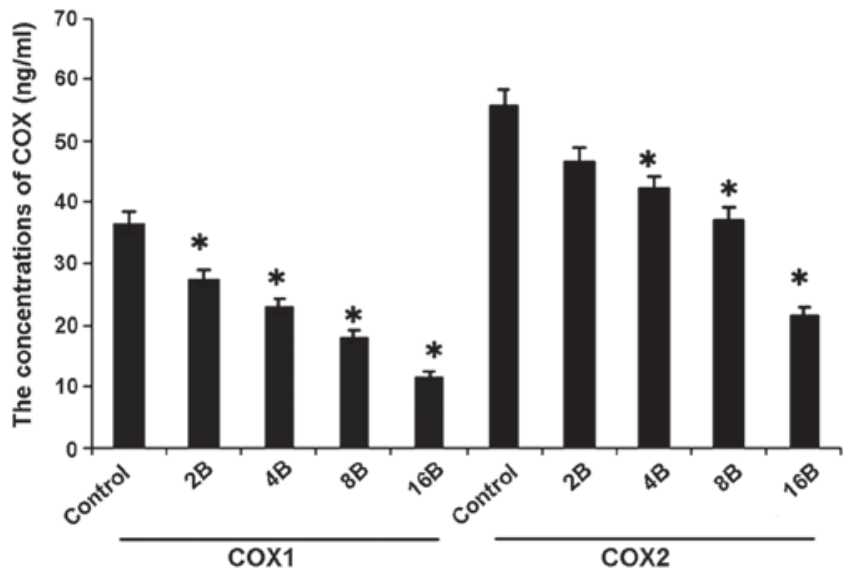

Figure 7. ELISA analyses show that blueberries reduce protein levels of COX-1 and COX-2 of ovarian cancer in a mouse model. The mice were treated with $0,2,4,8$ or $16 \mathrm{mg} / \mathrm{ml}$ blueberry juice. The protein levels of COX-1 and COX-2 were measured subsequent to a 14-day culture. The data represent the results of 5 independent experiments and are presented as the mean \pm standard deviation. ${ }^{*} \mathrm{P}<0.05$ compared with $0 \mu \mathrm{g} / \mathrm{ml}$ blueberry. COX, cyclooxygenase, Control, without transfection or blueberry; $2 \mathrm{~B}, 2 \mathrm{mg}$ blueberry; 4B, $4 \mathrm{mg}$ blueberry; 8B, $8 \mathrm{mg}$ blueberry; 16B, $16 \mathrm{mg}$ blueberry.

\section{Discussion}

$\mathrm{OC}$ is a common cause of female mortality worldwide and blueberry therapy has been identified to be effective in the treatment of various types of carcinoma $(21,22,29)$. The theoretical benefit of blueberry juice as a salvage therapy is associated with the anti-inflammation capacity $(30,31)$ and ability to prevent the progression of various types of cancer $(32,33)$. However, the molecular mechanism of the inhibition of OC by blueberries remains unclear. Therefore, the present study aimed to address the issue, and revealed a significant result with respect to the use of blueberry juice for the treatment of $\mathrm{OC}$ in the BALB/c nude mouse model. Based on the aforementioned information, the present study firstly reported the molecular mechanisms for the inhibition of OC by blueberry, revealing that a suitable dosage of blueberry juice decreases the expression of COX-1 and COX-2, which are 2 biomarkers for the development of $\mathrm{OC}$. The aforementioned findings suggest that blueberry juice decreases the levels of COX-1 and COX-2, and inhibits the progression of OC. Furthermore, detecting the levels of COX-1 and COX-2 aid in the prediction of patients at risk for OC, as reported in previous studies $(24,34)$. Blueberry juice therapy may therefore provide a non-pharmaceutical treatment for patients with OC.

Regarding the promotion of the growth of SKOV3 by COX-1 and COX-2, the issue that growth rate may be affected by other molecules must be considered. Therefore, the present study investigated the overexpression and gene silencing of COX-1 and COX-2, revealing that when COX-1 and COX-2 were overexpressed without blueberry treatment, the growth rate of SKOV3 reached the highest level of the present study. By contrast, when COX-1 and COX-2 were silenced by RNAi and treated with blueberry juice at a high concentration, the growth rate of SKOV3 reached the lowest level of the present study (Fig. 1). Furthermore, blueberry juice reduces the levels of COX-1 and COX-2 in a dose-dependent way (Figs. 2-5). The aforementioned results suggest that COX-1 and COX-2 
promote the growth of $\mathrm{OC}$ while blueberry juice inhibits the development of OC by downregulating the levels of COX-1 and COX-2.

There were certain limitations of the present study. For example, blueberry juice possesses a number of different components, which were not separated or purified to identify the more effective agents for the treatment of OC. The functions of blueberries are not unique and more functions require exploration; a limitation of the present study is that the precise mechanism of blueberries with respect to the downregulation of the levels of COX-1 and COX-2 remains unknown. The components of blueberry juice should be analyzed in detail, which may offer information to understand the exact molecular mechanisms for the role of blueberry juice in the therapy of OC.

The present study revealed that the efficacy of using blueberries to treat $\mathrm{OC}$ is significant. To make full use of blueberries, all associated molecular mechanisms should be investigated to maximize the potential benefit of blueberries and minimize the risk of side effects. The present study demonstrated that the concentration at which blueberries exhibit significant inhibition is $16 \mathrm{mg} / \mathrm{ml}$ for OC cells. At this concentration, blueberries effectively treated the OC models. The results of the present study provide information for subsequent clinical trials and may be beneficial to utilize blueberries, effectively and safely, as a non-pharmaceutical OC therapy. Considering the effectiveness and safety of blueberries, a larger sample size and long-term follow-up test in a larger sample of mice is recommended.

In conclusion, blueberries have been demonstrated to be effective and safe in controlling the size of $\mathrm{OC}$ in a dose-dependent way. Blueberries inhibit the proliferation of OC cells by downregulating the levels of COX-1 and COX-2 . The present study established animal models of OC by the injection of SKOV3 cells into nude mice. Blueberry (400 mg daily) consumption decreased tumor size significantly in the mice with OC compared with the controls without blueberry treatment $(\mathrm{P}<0.05)$. The results of the present study suggest that blueberries should be developed as a potential non-pharmaceutical therapy for OC.

\section{References}

1. Donovan KA, Donovan HS, Cella D, Gaines ME, Penson RT, Plaxe SC, von Gruenigen VE, Bruner DW, Reeve BB and Wenzel L: Recommended patient-reported core set of symptoms and quality-of-life domains to measure in ovarian cancer treatment trials. J Natl Cancer Inst 106: pii: dju128, 2014.

2. Chen $\mathrm{CH}$, Chiu LH, Chan $\mathrm{C}$ and Liu WM: Management of ovarian cancer in 14th gestational week of pregnancy by robotic approach with preservation of the fetus. Gynecol Obstet Invest 80: 139-144, 2015.

3. Yang XJ, Zheng FY, Xu YS and Ou RY: Ovarian cancer initially presenting with isolated ipsilateral superficial inguinal lymph node metastasis: A case study and review of the literature. J Ovarian Res 7: 20, 2014.

4. Shim SH, Kim DY, Seo MJ, Lee SW, Park JY, Lee JJ, Kim JH, Kim YM, Kim YT and Nam JH: Preoperative fluorine 18 fluorodeoxyglucose tumoral uptake ratio between upper and lower abdomen in primary advanced-stage ovarian cancer. Int $\mathbf{J}$ Gynecol Cancer 23: 1383-1392, 2013.

5. Peng X, Wang P, Li S, Zhang G and Hu S: Randomized clinical trial comparing octreotide and scopolamine butylbromide in symptom control of patients with inoperable bowel obstruction due to advanced ovarian cancer. World J Surg Oncol 13: 50, 2015.
6. Mir MC, Stephenson AJ, Grubb RL III, Black A, Kibel AS and Izmirlian G: Predicting risk of bladder cancer using clinical and demographic information from prostate, lung, colorectal, and ovarian cancer screening trial participants. Cancer Epidemiol Biomarkers Prev 22: 2241-2249, 2013.

7. Nagano H, Muraoka M and Takagi K: Recurrent ovarian cancer with multiple lymph nodes metastases successfully treated with lymphadenectomy as secondary cytoreductive surgery: A case report. Int J Surg Case Rep 5: 412-415, 2014.

8. Bacalbasa $\mathrm{N}$ and Popescu I: Ovarian cancer liver metastases-should we apply the principle of optimal cytoreduction to the liver? A review. Hepatogastroenterology 62: 355-357, 2015.

9. Ferlay J, Soerjomataram I, Dikshit R, Eser S, Mathers C, Rebelo M, Parkin DM, Forman D and Bray F: Cancer incidence and mortality worldwide: Sources, methods and major patterns in GLOBOCAN 2012. Int J Cancer 136: E359-E386, 2015.

10. Frampton JE: Olaparib: A review of its use as maintenance therapy in patients with ovarian cancer. BioDrugs 29: 143-150, 2015.

11. Ledermann J, Harter P and Gourley C: Correction to Lancet Oncol 2014; 15: 856. Olaparib maintenance therapy in patients with platinum-sensitive relapsed serous ovarian cancer: A preplanned retrospective analysis of outcomes by BRCA status in a randomised phase 2 trial. Lancet Oncol 16: e158, 2015.

12. Fu X, Zhang Y, Wang X, Chen M, Wang Y, Nie J, Meng Y and Han W: Low dose decitabine combined with taxol and platinum chemotherapy to treat refractory/recurrent ovarian cancer: An open-label, single-arm, Phase I/II study. Curr Protein Pept Sci 16: 329-336, 2015.

13. McGrail DJ, Khambhati NN, Qi MX, Patel KS, Ravikumar N, Brandenburg CP and Dawson MR: Alterations in ovarian cancer cell adhesion drive taxol resistance by increasing microtubule dynamics in a FAK-dependent manner. Sci Rep 5: 9529, 2015.

14. Pettitt SJ, Rehman FL, Bajrami I, Brough R, Wallberg F, Kozarewa I, Fenwick K, Assiotis I, Chen L, Campbell J, et al: A genetic screen using the PiggyBac transposon in haploid cells identifies Parp1 as a mediator of olaparib toxicity. PLoS One 8: e61520, 2013.

15. McNeil EM, Ritchie AM and Melton DW: The toxicity of nitrofuran compounds on melanoma and neuroblastoma cells is enhanced by Olaparib and ameliorated by melanin pigment. DNA Repair (Amst) 12: 1000-1006, 2013.

16. Bouquet W, Ceelen W, Adriaens E, Almeida A, Quinten T, De Vos F, Pattyn P, Peeters M, Remon JP and Vervaet C: In vivo toxicity and bioavailability of Taxol and a paclitaxel/beta-cyclodextrin formulation in a rat model during HIPEC. Ann Surg Oncol 17: 2510-2517, 2010.

17. Arany I, Clark JS, Reed D, Szabo I, Ember I and Juncos LA: The role of p66shc in taxol- and dichloroacetic acid-dependent renal toxicity. Anticancer Res 33: 3119-3122, 2013.

18. Sun CC, Bodurka DC, Weaver CB, Rasu R, Wolf JK, Bevers MW, Smith JA, Wharton JT and Rubenstein EB: Rankings and symptom assessments of side effects from chemotherapy: Insights from experienced patients with ovarian cancer. Support Care Cancer 13: 219-227, 2005.

19. Faria A, Pestana D, Teixeira D, de Freitas V, Mateus N and Calhau C: Blueberry anthocyanins and pyruvic acid adducts: Anticancer properties in breast cancer cell lines. Phytother Res 24: 1862-1869, 2010.

20. Zu XY, Zhang ZY, Zhang XW, Yoshioka M, Yang YN and Li J: Anthocyanins extracted from Chinese blueberry (Vaccinium uliginosum L.) and its anticancer effects on DLD-1 and COLO205 cells. Chin Med J (Engl) 123: 2714-2719, 2010.

21. Kanaya N, Adams L, Takasaki A and Chen S: Whole blueberry powder inhibits metastasis of triple negative breast cancer in a xenograft mouse model through modulation of inflammatory cytokines. Nutr Cancer 66: 242-248, 2014.

22. Jeyabalan J, Aqil F, Munagala R, Annamalai L, Vadhanam MV and Gupta RC: Chemopreventive and therapeutic activity of dietary blueberry against estrogen-mediated breast cancer. J Agric Food Chem 62: 3963-3971, 2014.

23. Thuresson ED, Lakkides KM and Smith WL: PGG2, 11R-HPETE and $15 \mathrm{R} / \mathrm{S}$-HPETE are formed from different conformers of arachidonic acid in the prostaglandin endoperoxide $\mathrm{H}$ synthase- 1 cyclooxygenase site. Adv Exp Med Biol 507: 67-72, 2002.

24. Wilson AJ, Fadare O, Beeghly-Fadiel A, Son DS, Liu Q, Zhao S, Saskowski J, Uddin MJ, Daniel C, Crews B, et al: Aberrant over-expression of COX-1 intersects multiple pro-tumorigenic pathways in high-grade serous ovarian cancer. Oncotarget 6: 21353-21368, 2015. 
25. Lin Y, Cui M, Xu T, Yu W and Zhang L: Silencing of cyclooxygenase-2 inhibits the growth, invasion and migration of ovarian cancer cells. Mol Med Rep 9: 2499-2504, 2014.

26. Hui X, Chen H, Zhang S, Ma X, Wang X and Huang B: Antitumor activities of recombinant human interferon (IFN)- $\lambda 1$ in vitro and in xenograft models in vivo for colon cancer. Cancer Lett 311: 141-151, 2011.

27. Livak KJ and Schmittgen TD: Analysis of relative gene expression data using real-time quantitative PCR and the 2(-Delta Delta C(T)) method. Methods 25: 402-408, 2001.

28. Yang WL, Roland IH, Godwin AK and Xu XX: Loss of TNF-alpha-regulated COX-2 expression in ovarian cancer cells. Oncogene 24: 7991-8002, 2005.

29. Montales MT, Rahal OM, Kang J, Rogers TJ, Prior RL, Wu X and Simmen RC: Repression of mammosphere formation of human breast cancer cells by soy isoflavone genistein and blueberry polyphenolic acids suggests diet-mediated targeting of cancer stem-like/progenitor cells. Carcinogenesis 33: 652-660, 2012

30. Esposito D, Chen A, Grace MH, Komarnytsky S and Lila MA: Inhibitory effects of wild blueberry anthocyanins and other flavonoids on biomarkers of acute and chronic inflammation in vitro. J Agric Food Chem 62: 7022-7028, 2014.
31. Paulis G, Cavallini G, Giorgio GD, Quattrocchi S, Brancato T and Alvaro R: Long-term multimodal therapy (verapamil associated with propolis, blueberry, vitamin $\mathrm{E}$ and local diclofenac) on patients with Peyronie's disease (chronic inflammation of the tunica albuginea). Results of a controlled study. Inflamm Allergy Drug Targets 12: 403-409, 2013.

32. Ge I, Rudolph A, Shivappa N, Flesch-Janys D, Hébert JR and Chang-Claude J: Dietary inflammation potential and postmenopausal breast cancer risk in a German case-control study. Breast 24: 491-496, 2015.

33. Khachatryan $\mathrm{N}$ and Kempen JH: Immunosuppressive therapy and cancer risk in ocular inflammation patients: Fresh evidence and more questions. Ophthalmology 122: 219-221, 2015.

34. Magnowska M, Zaborowski M, Surowiak P, Nowak-Markwitz E, Zabel M and Spaczyński M: COX-2 expression pattern is related to ovarian cancer differentiation and prognosis, but is not consistent with new model of pathogenesis. Ginekol Pol 85: 335-341, 2014. 